\title{
Pancreaticoduodenectomy With Arterial Approach of Total Mesenteric Resection of the Pancreas for Pancreatic Head Cancer
}

\author{
Futian Dua, b, Xin Wang ${ }^{\mathrm{a}}$, Hongfeng Lin ${ }^{\mathrm{a}}$, Xuelin Zhao ${ }^{\mathrm{a}}$
}

\begin{abstract}
Background: This study aims to observe and analyze the clinical efficacy of the pancreaticoduodenectomy (PD) with total mesopancreas excision $(\mathrm{TMpE})$ via the artery approach for carcinoma of head of the pancreas.
\end{abstract}

Methods: From October 2015 to October 2016, 60 patients with pancreatic head cancer were enrolled in this study. Twenty-eight patients were treated with PD with TMpE via the artery approach (group A), while 32 patients were treated with PD alone (group B) in our hospital. The clinical data of the patients were retrospectively collected, including intra-operative evaluation index, R0 resection rate of postoperative pathological specimens, postoperative complications, and the tumor recurrence time was observed after operation (at third, sixth, and 12th months). Clinical efficacy of PD with TMpE via the artery approach was evaluated between the two groups.

Results: There was no significant difference in the operation time or perioperative death between the two groups $(\mathrm{P}>0.05)$. Postoperative specimen pathology showed that there was a statistically significant difference in the $\mathrm{R} 0$ resection rate between the two groups $(\mathrm{P}<0.05)$. There was no significant difference in the incidence of postoperative complications between the two groups $(\mathrm{P}>0.05)$. Tumor recurrence rate at month 3 post operation was $0(0 / 28)$ in group A and $3.13 \%$ $(1 / 32)$ in group B. There was no significant difference in the 6-month recurrence rate $(\mathrm{P}>0.05)$. Postoperative recurrence rate in group $A$ was significantly lower than that in group $\mathrm{B}$ at month $12(\mathrm{P}<0.05)$.

Conclusions: The PD with $\mathrm{TMpE}$ via the artery approach treatment of pancreatic head cancer can reduce the amount of intra-operative bleeding and save the operation time without increasing postoperative complications. It provides effective technical support for combined vascular anastomosis in the treatment of pancreatic head cancer with venous system invasion, and ensures the safety of operation. Moreo-

Manuscript submitted September 16, 2019, accepted September 28, 2019

aDepartment of Hepatobiliary Surgery, Weifang People's Hospital, Weifang, Shandong 261000, China

bCorresponding Author: Futian Du, Department of Hepatobiliary Surgery, Weifang People's Hospital, Weifang, Shandong 261000, China.

Email:dft641016@163.com

doi: https://doi.org/10.14740/gr1225 ver, this procedure can improve the $\mathrm{R} 0$ resection rate and reduce the recurrence rate in the near future. As a safe, effective and feasible surgical method for the treatment of pancreatic head cancer, it can be widely used in clinical practice.

Keywords: Pancreaticoduodenectomy; Arterial priority approach; Total mesopancreas excision; Pancreatic head cancer

\section{Introduction}

Pancreatic head cancer has the characteristics of rapid development, difficulty in early detection, high degree of malignancy, low resection rate and poor prognosis $[1,2]$. With abdominal pain in the upper abdomen, yellowing of the skin mucosa, itching of the skin, weight loss, and so on, patients often have progressed to the advanced stage of pancreatic head cancer when they come to the hospital. In recent years, the incidence of pancreatic head cancer has increased significantly at home and abroad. After Whipple et al proposed the operation of pancreaticoduodenectomy (PD) in 1935, this procedure has become the standard procedure for the treatment of pancreatic head cancer [3].

Although PD is the only radical surgical procedure for the treatment of pancreatic head cancer, the long-term recurrence rate and long-term survival rate of patients with pancreatic head cancer after PD are mainly dependent on radical R0 resection [4]. Related literature reports that the mortality of patients with pancreatic head cancer after PD is maintained at about $5 \%$ [5]. Due to the difficulty of PD operation, high technical requirements, complicated surgical methods, and short postoperative recurrence time, scholars at home and abroad have put forward various surgical operation techniques, and reduced PD by changing the way of PD and the operation mode. They try to improve $\mathrm{R} 0$ resection rate, reduce the recent recurrence rate of patients after PD, and increase the long-term survival rate of postoperative patients, such as through arterial priority and total mesopancreas excision (TMpE) resection.

In this paper we summarized the clinical data of 28 patients who underwent PD resection with TMpE from October 2015 to October 2016 in the Department of Hepatobiliary Surgery of Weifang People's Hospital for pancreatic cancer. Compared with 32 patients with conventional $\mathrm{PD}$, it is proved that the 

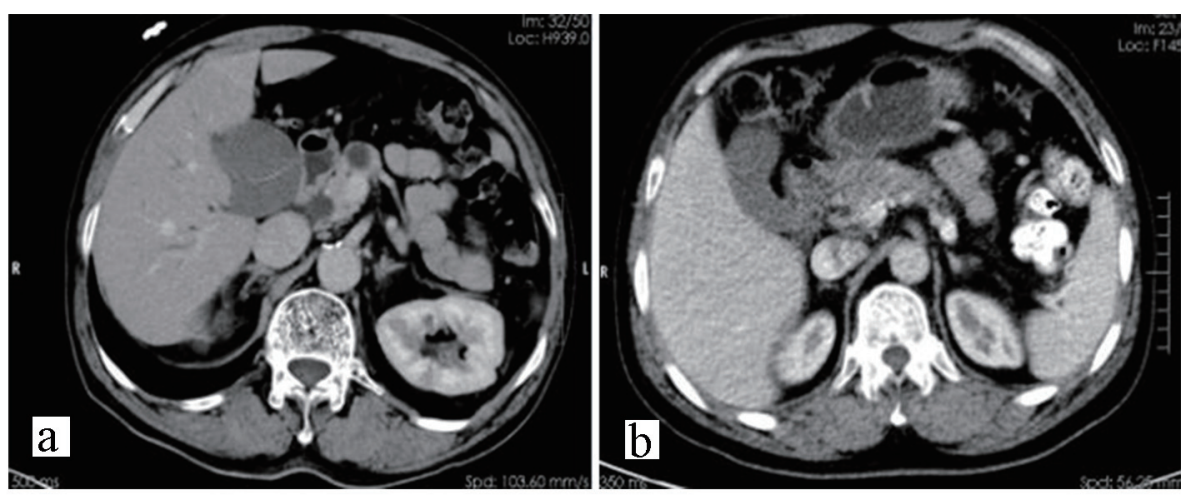

Figure 1. Preoperative computed tomography findings of pancreatic head cancer without invasion of the venous system (a) or with invasion of the venous system (b).

arterial priority approach TMpE resected PD ensures the safety of surgery, and this procedure has no significant effect on the incidence of postoperative complications compared with traditional PD, and can reduce the rate of recent tumor recurrence. $\mathrm{TMpE}$ is a safe, effective and feasible surgical method for the treatment of pancreatic head cancer. It provides further clinical reference data and theoretical basis for the treatment of pancreatic head cancer with TMpE resected by arterial priority.

\section{Materials and Methods}

\section{Study design}

This study included 60 patients with pancreatic head cancer. They were divided into two groups. Group A included patients with pancreatic head cancer who underwent PD treatment with $\mathrm{TMpE}$ resection of the arterial priority approach. There were 28 cases, including 16 men and 12 women, aged 44 - 66 years, with an average age of 57 years. Group B included patients with pancreatic head cancer who underwent traditional PD therapy alone. There were 19 men and 13 women, aged 48 - 69 years, with an average age of 55 years.

All data were from 32 patients with pancreatic head cancer who were treated in the Hepatobiliary Surgery Department of Weifang People's Hospital from October 2015 to October 2016. The clinical data of the patients were retrospectively collected, including intra-operative evaluation index (operative time, intra-operative blood loss), R0 resection rate of postoperative pathological specimens, postoperative complications (postoperative bleeding, pancreatic leakage, biliary fistula (BF), infection, delayed gastric emptying, diarrhea), and the tumor recurrence time was observed after operation (at third, sixth, and 12th months). This study was approved by the Research Ethics Board (REB) of the hospital.

\section{Observation indicators}

As for perioperative observation indicators, both groups recorded the operation time and intra-operative blood loss; post- operatively attention was paid to complications (postoperative infection, postoperative bleeding, pancreatic fistula $(\mathrm{PF}), \mathrm{BF}$, delayed gastric emptying (DGE), diarrhea and other complications), monitoring and treatment of common adverse reactions after surgery.

As for the post-discharge observation indicators, after the patients were discharged from the hospital, they were examined at the third, sixth, and 12th months. The upper abdominal computed tomography scan + intensive, carbohydrate antigen 199 (CA199) was observed, and the tumor recurrence and metastasis were observed (Fig. 1). After discharge, patients' condition changed and corresponding complications were followed up.

\section{Treatment procedures}

Group A: PD with total mesenteric resection of the pancreas

The patients were placed in the supine position. After the general anesthesia was intubated, the right L-shaped incision was made into the abdominal cavity (AC). The liver and AC were routinely examined for metastasis. After the liver and intraabdominal metastases were removed, the enlarged Kocker incision was made. At duodenal side peritoneum, we carefully distracted the duodenum and the posterior tissue of the pancreatic head, free until the front of the abdominal aorta (AA), inferior vena cava (IVC) and left kidney. After the vein was fully exposed, particular attention should be paid to the cleaning of connective tissue between AA and IVC. If the superior mesenteric artery (SMA) was invaded by the tumor, the pancreaticoduodenal artery was separated from the SMA root, and then the pancreaticoduodenal artery was ligated and disconnected. The SMA was suspended with a silicone tube, so that the small branch of the SMA was ligated, and the SMA was further dissected to the border between the horizontal and the ascending parts of the duodenum. During this process, attention was paid to the right hepatic artery that may originate from the SMA (right branch). If the SMA was not invaded by the tumor, the pancreatic mesangium attached to the SMA could be cut, the tissues such as fat and lymph in front of the SMA and the right side were cleaned, the connective tissue between the 


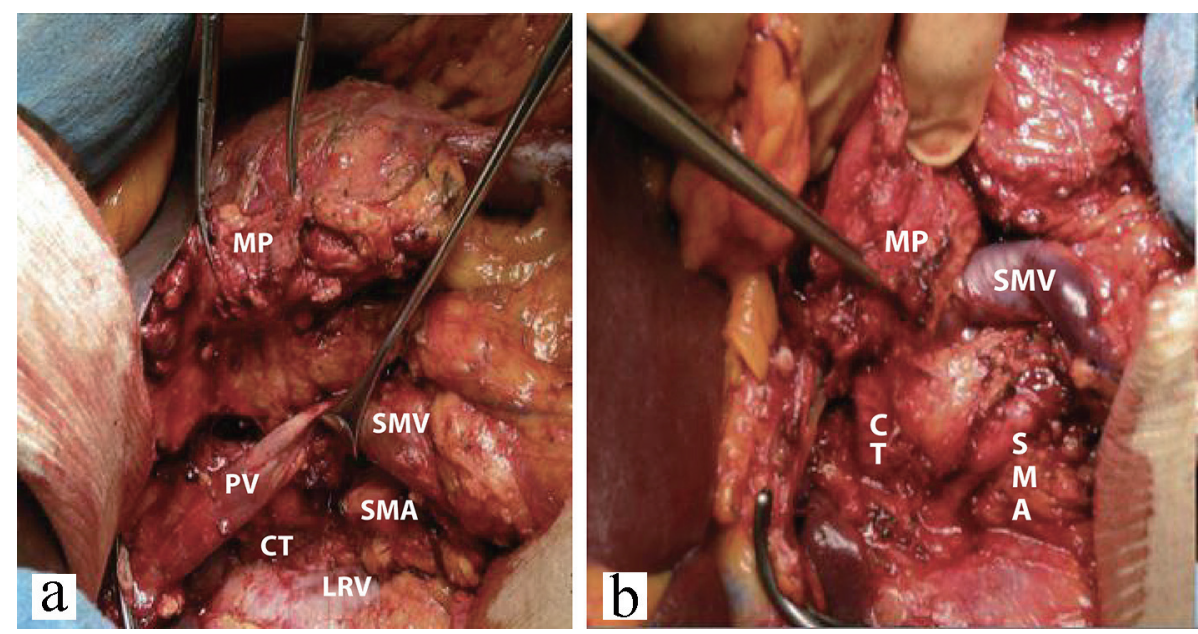

Figure 2. PD with TMpE resection $(a, b)$. Surgical procedures are described in the Methods section. PD: pancreaticoduodenectomy; TMpE: mesopancreas excision; SMV: superior mesenteric vein; SMA: superior mesenteric artery; PV: portal vein; CT: coeliacus truncus; LRV: left renal vein; MP: mesopancreas.

SMA and the superior mesenteric vein (SMV) was swept, and the posterior wall of the SMV was exposed. Then the gastric collateral ligament was opened, and the omentum was disconnected from the inside of the gastric retinal vascular arch. After the stomach was separated, the small omental sac was opened, and the left edge of the hepatoduodenal ligament was directly reached. The first and third groups of lymph nodes were removed. After exposing and suspending the common hepatic artery at the upper edge of the pancreas, the gastroduodenal artery was found on the common hepatic artery of the pancreas, and then the gastroduodenal artery was ligated and cut off so that the hepatic artery along the common hepatic artery can be dissected. The right hepatic artery was ligated to the right gastric artery, the common hepatic artery was skeletal, the $\mathrm{AC}$ of the root was directly removed, and the lymph nodes on the AC were cleaned. The ligaments of the hepatic artery and its branches were connected to the hepatic artery, and the connective tissue on the left edge of the portal vein (PV) was removed. The hepatic duct was transected, the distal end of the common hepatic duct was ligated, and the connective tissue on the right side of the PV was gradually separated into the duodenum to the upper edge of the pancreas. We cleared the upper edge of the pancreas and the connective tissue behind the PV, and then completed the lymph node dissection. The jejunum was cut $10-15 \mathrm{~cm}$ from the ligament of the flex. The anterior wall of PV and SMV at the upper and lower edges of the pancreas was exposed, and the pancreatic neck was ligated and cut in front of it. The mesenteric membrane of the pancreas was dissected, and the anterior wall of AA was exposed. The anterior wall of AA was dissected up to $\mathrm{AC}$, the connective tissue on the surface of $\mathrm{AC}$ was cut, the pancreatic mesangium attached to $\mathrm{AC}$ was cut, and the lymphatic tissue on the right side of AC was cleaned. If the SMV and PV were not invaded by the tumor, the branches of the PV and SMV can be ligated from the top to the bottom of the pancreatic head and from the right to the left. If the SMV and PV were suspected to be invaded, the SMV and PV can be pretreated. After the blocking band was placed, the branches were ligated and disconnected.
The SMV and PV can be safely stripped only by the pressurereceiving person. The SMV and PV invaded can be anastomosed by the invaded vascular resection, and the whole PD specimen has been completely removed. The lymph nodes in the SMA root, the AC root and the AA were then further cleaned. Finally, pancreaticojejunostomy, gastrointestinal anastomosis, and biliary anastomosis were performed. After the AC was fully washed, two abdominal drainage tubes were placed at the anastomosis of the pancreas and the anastomosis of the biliary and intestines (Fig. 2a).

\section{Group B: traditional PD}

The patients were placed in the supine position. After the tracheal intubation was performed, the right L-shaped incision was made into the AC. The abdomen, the liver and the $\mathrm{AC}$ were routinely examined for metastasis. After the liver and intraabdominal metastases were removed, the Kocker incision was performed and the 12 incision was made. We referred to the intestinal side of the peritoneum to detect whether PV/SMV was invaded by the tumor of the head of the pancreas. For patients who had not been invaded, the distal stomach and the common hepatic artery were dissected, the gastric duodenal artery was isolated, ligated, and the duodenal artery was removed, and then the gallbladder, the common hepatic duct, and the hepatoduodenal ligament were separated. We performed ligation, disconnection of the pancreatic neck, ligation, disconnection of the pancreatic head and pancreatic uncinate process and the vein branch between PV and SMV, cut the jejunum 10 - 15 $\mathrm{cm}$ from the flexor ligament. At this time, only the uncinate part of the pancreas was connected with the SMA, and the fibrous sheath of the SMA was cut longitudinally. After a little separation, the uncinate membrane was clearly revealed, and the occlusion, cutting, and ligation were gradually performed from top to bottom. With the tissue connection between the SMA and the pancreas and the arterial blood supply, attention was paid to the ligation of the pancreaticoduodenal artery 
Table 1. Intra-Operative Operation Time and Bleeding Volume Observation (Mean \pm SEM, $n=28$ in Group A, $n=32$ in Group B)

\begin{tabular}{lll} 
Groups & Operation time (min) & $\begin{array}{l}\text { Intra-operative } \\
\text { blood loss }(\mathbf{m L})\end{array}$ \\
\hline Group A & $321.43 \pm 43.03$ & $366.07 \pm 109.76$ \\
Group B & $333.66 \pm 29.77$ & $557.81 \pm 119.89$ \\
T value & -1.262 & -6.427 \\
P value & 0.213 & $<0.001^{*}$ \\
\hline
\end{tabular}

Group A is the PD group with TMpE resection, and group B is the traditional PD group. SEM: standard error of mean; PD: pancreaticoduodenectomy; TMpE: total mesopancreas excision. ${ }^{*} P<0.001$.

alone; at this time the specimen was completely removed for patients with PV/SMV aggression; after rupturing the neck of the pancreas, the jejunum and uncinate membrane were cut off, and then the vessel of the invaded vein was resected and reconstructed. Finally, the operation was the same as group A (Fig. 2b).

\section{Statistical analysis}

The collected data were statistically analyzed by SPSS 19.0 statistical software package. All the measurement data were represented by (mean \pm standard error of mean (SEM)). The $t$-test was used to compare the two groups of samples, and the Chisquare test and Fisher exact test were used to compare the count data. $\mathrm{P}<0.05$ was considered statistically significant difference.

\section{Results}

All patients in both groups had a smooth operation, and no deaths occurred during or after surgery. For group A, preoperative imaging examination revealed 11 patients with suspected $\mathrm{PV} / \mathrm{SMV}$ infringement. For group B, preoperative imaging showed nine patients with suspected PV/SMV infringement. All 20 patients were treated. It was found that the tumor of the head of the pancreas was closely related to PV/SMV, and it was not easy to be stripped. Considering the possibility of invasion, there were two cases of PV invasion in group A, nine cases of invasion of SMV, one case of invasion of PV in group $\mathrm{B}$, and eight cases of invasion of SMV. For example, 20 patients underwent intra-operative total anastomosis with acupuncture site. The relevant efficacy indicators of groups A and $\mathrm{B}$ were analyzed as follows.

\section{Intra-operative operation time and bleeding volume obser-} vation

The operation time of group A was $321.43 \pm 43.03 \mathrm{~min}$, and the intra-operative blood loss was $366.07 \pm 109.76 \mathrm{~mL}$; the operation time of group B was $333.66 \pm 29.77 \mathrm{~min}$, and the intra-operative blood loss was $557.81 \pm 119.89 \mathrm{~mL}$. There was no significant difference in operation time between the two
Table 2. R0 Resection Rate of Postoperative Specimen Pathology ( $n=28$ in Group A, $n=32$ in Group B)

\begin{tabular}{lll}
\hline Groups & R0 cut number & R0 resection rate \\
\hline Group A (24/28) & 24 & $85.71 \%$ \\
Group B (20/32) & 20 & $62.50 \%$ \\
X $^{2}$ & 4.115 & \\
P value & $0.042 *$ & \\
\hline
\end{tabular}

Group A is the PD group with TMpE resection, and group B is the traditional PD group. PD: pancreaticoduodenectomy; TMpE: total mesopancreas excision. ${ }^{*} \mathrm{P}<0.05$.

groups $(\mathrm{P}>0.05)$, while the difference in intra-operative blood loss between the two groups was statistically significant $(\mathrm{P}<$ 0.05) (Table 1).

\section{$\mathrm{R} 0$ resection rate of postoperative specimen pathology}

The $\mathrm{R} 0$ resection rate was $85.71 \%(24 / 28)$ in group A and $62.50 \%$ (20/32) in group B. There was significant difference in the R0 resection rate between the two groups $(\mathrm{P}<0.05)$ (Table 2$)$.

\section{Comparison of postoperative complications}

The incidence of postoperative complications in group A was $25.00 \%$ (7/28), including one case of infection, zero case of postoperative bleeding, two cases of PF, zero case of BF, three cases of diarrhea, one case of DGE; the incidence rate was $28.13 \%$ (9/32), including two cases of infection, one case of postoperative hemorrhage, two cases of PF, two cases of BF, two cases of diarrhea and two cases of DGE. There was no difference in the incidence of postoperative complications between the two groups. No statistical significance was observed between the two groups $(\mathrm{P}>0.05)$ (Table 3$)$. Postoperative complications in both groups were cured by conservative medical treatment.

\section{Postoperative tumor recurrence}

The tumor recurrence rate at month 3 after operation was $0(0 / 28)$ in group A and 3.57\% (1/32) in group B. The recurrence rate of tumor at month 6 after operation was $3.57 \%(1 / 28)$ in group A and $12.50 \%(4 / 32)$ in group B. The recurrence rate at month 12 postoperatively was $7.14 \%(2 / 28)$ in group A, and $28.13 \%$ $(9 / 32)$ in group B. There was no significant difference at months 3 and 6 post operation in the recurrence rate between the two groups $(\mathrm{P}>0.05)$; however, there was a significant difference at month 12 post operation between them $(\mathrm{P}<0.05)$ (Table 4$)$.

\section{Discussion}

Our study and others showed that the PD with TMpE via the artery approach may be applied as a standard care for the treat- 
Table 3. Comparison of Postoperative Complications ( $n=28$ in Group A, $n=32$ in Group B)

\begin{tabular}{lllllllll}
\hline Groups & Total & Incidence rate & Infection & $\begin{array}{l}\text { Postoperative } \\
\text { bleeding }\end{array}$ & Pancreatic fistula & Biliary fistulae & $\begin{array}{l}\text { Diarrhea } \\
\text { Demptying }\end{array}$ \\
\hline Group A & 7 & $25.00 \%(7 / 28)$ & 1 & 0 & 2 & 0 & 3 & 1 \\
Group B & 9 & $28.13 \%(9 / 32)$ & 2 & 1 & 2 & 0 & 2 & 2 \\
$\mathrm{X}^{2}$ & 0.075 & & & & & & \\
P value & 0.785 & & & & & & \\
\hline
\end{tabular}

Group A is the PD group with arterial priority approach TMpE resection, and group B is the traditional PD group. PD: pancreaticoduodenectomy; TMpE: total mesopancreas excision.

ment of pancreatic head cancer. The advantages of PD with preferential arterial approach include reduction of unnecessary surgical injury, surgical bleeding and operation time, and early recurrence rate, as well as consistency with the "non-contact" principle of tumor resection.

First of all, according to the 2010 National Comprehensive Cancer Network (NCCN) guidelines of China: SMA violation $>180^{\circ}$ is an unresectable sign of pancreatic head cancer, while PV/SMV invasion can be reconstructed; SMA $<180^{\circ}$ is borderline resectable, such as Achieving R0 resection will benefit the patient. The priority of the arterial approach to PD is to perform SMA exploration, which can be used to determine whether SMA is invaded by the tumor of the head of the pancreas and decide whether to continue the operation and reduce unnecessary surgical trauma.

Secondly, this procedure can reduce surgical bleeding and shorten the operation time: the PD with preferential arterial priority preferentially disconnects the arterial blood supply from the head of the pancreas. At the same time, the PD with preferential arterial approach can temporarily avoid the anterior wall of free PV and SMV, directly. The left side of the PV, SMV vascular area establishes a safe area for cutting the neck of the pancreas, thereby reducing arteriovenous hemorrhage and shortening the operation time. Especially for patients with suspected PV/SMV violations, it is the preferred surgical method. Dumitrascu found that PD through the preferential approach of the arteries can significantly shorten the operation time and greatly reduce the intra-operative blood loss of PD [6].

Thirdly, the traditional postoperative retroperitoneal rate of PD is high, and the PD with preferential arterial approach achieves complete resection of the right nerve, lymph, and adipose tissue with SMA and celiac trunk as the axis. The pancre-

Table 4. Postoperative Tumor Recurrence $(n=28$ in Group A, $\mathrm{n}=32$ in Group B)

\begin{tabular}{llll} 
Groups & $\begin{array}{l}\text { Three months } \\
\text { after surgery }\end{array}$ & $\begin{array}{l}\text { Six months } \\
\text { after surgery }\end{array}$ & $\begin{array}{l}\text { Twelve months } \\
\text { after surgery }\end{array}$ \\
\hline Group A & 0 & 1 & 2 \\
Group B & 1 & 4 & 9 \\
$X^{2}$ & & 0.609 & 4.391 \\
P value & 1 & 0.435 & $0.036^{*}$ \\
\hline
\end{tabular}

Group A is the PD group with TMpE resection, and group B is the traditional PD group. PD: pancreaticoduodenectomy; TMpE: total mesopancreas excision. ${ }^{*} \mathrm{P}<0.05$. atic total mesenteric resection is beneficial to achieve R0 resection of the retroperitoneal margin, reducing the recurrence of the retroperitoneal margin of the patient. The results of the $\mathrm{R} 0$ resection rate obtained in this study are consistent with this.

Lastly, it is more consistent with the "non-contact" principle of tumor resection. PD selection with preferential arterial approach reveals SMA, SMV from the lateral rear of the head of the pancreatic head tumor, disconnects the neck of the pancreas, and separates the uncinate process. Not only is it easier to expose the surgical field, but the suspension and pulling of the blood vessel can greatly reduce the extrusion and pulling of the tumor of the pancreatic head when the uncinate process is performed in the PD, and complete the "non-contact" resection of the tumor. Especially for patients with suspected violations of PV and SMV, the arterial priority approach satisfies the whole resection of the pancreatic head tumor under the principle of "non-contact" and avoids the spread of tumor cells through the PV. Gall et al reported other patients in the PD specimens were excised from the PV for microscopic tumor cell detection [7]. It was found that $83 \%$ of patients with traditional PD had access to tumor cells in the PV, while those in the non-contact group did not. The survival periods were 13 and 16.7 months, respectively.

There are several limitations of traditional PD. Currently, pancreatic cancer has become the top 10 cancers associated with cancer-related death [8]. PD is the only effective treatment for pancreatic head cancer. In more than 80 years since its inception, with the continuous research and development of clinical operations, it has been gradually found that traditional PD has certain deficiencies and defects.

The traditional PD is to exclude the liver and AC spread first, and then to perform Kocher incision, check the pancreas, PV and SMV for invasion, metastasis; if the exploration found that PV and SMV have not been invaded by the tumor, then giving patients $\mathrm{PD}$ radical resection of pancreatic head cancer should be considered. In the whole process of exploration of the AC, peripancreas, PV and SMV, because the tumor of the head of the pancreas is repeatedly squeezed by the surgeon, it may cause the tumor cells to fall into the blood and cause the blood to spread and metastasize.

\section{Common complications and prevention of PD in total mes- enteric resection of the pancreas}

The mortality of patients with pancreatic head cancer after 
PD is maintained at around 5\% [5], but the incidence of early postoperative complications can be as high as $25-70 \%$ [9-11]. Combined with the relevant literature and a comprehensive analysis of postoperative complications in the two groups of patients in this study, the most common complications after PD were hemorrhage, PF, BF, infection, DGE, and so on. Arterial priority approach $\mathrm{TMpE}$ resection of $\mathrm{PD}$ in addition to common postoperative complications of PD also has a common complication of diarrhea; as reported in the literature [12], arterial priority approach $\mathrm{TMpE}$ resection of $\mathrm{PD}$ will increase the incidence of postoperative diarrhea.

The anatomy of the head of the pancreas is particularly suitable for the cancer. It is easy to involve the SMV or the $\mathrm{PV}$. The pancreatic cancer that invades SMV/PV has been regarded as a contraindication for surgery. In recent years, with the improvement of surgical techniques, PD combined with $\mathrm{SMV} / \mathrm{PV}$ resection has also become possible. Combined venous resection has not increased the incidence of perioperative complications, and can be obtained in the same way as standard surgery for the patients without venous involvement. Ravikumar et al also retrospectively analyzed the incidence and mortality of 230 patients with combined vascular resection of PD and 840 standard PD perioperative complications, and the difference between the two groups was not statistically significant [13]. Zhou et al collected data from 2,247 patients with pancreatic cancer who underwent pancreatic resection in Europe, America, and Asia. Among them, 661 patients underwent vascular resection. There was no significant difference in perioperative complication rate compared with patients without combined vascular resection [14].

In fact, pancreatic cancer invasion of the PV and/or SMV does not imply the existence of extensive post-peritoneal invasion and distant metastasis. The inability of the tumor to separate from the blood vessels does not imply that the blood vessels are violated. The latter may be simply because the tumor is close to the PV and/or the SMV. Tumor growth involves blood vessels and forms inflammatory adhesions. It is not necessarily an invasive behavior of the tumor, and is essentially different from "blood transfer" [15]. Yekebas et al reported 100 cases of pancreatic cancer patients treated with combined vascular resection, and the final pathology showed that only 77 cases were true tumor invasion [16]. Shimoda et al reported that only 29 cases of combined vascular resection in all cases showed tumor invasion of blood vessels [17]. Recent studies by Rehders et al have pointed out that the appearance of peripheral blood tumor cells is not associated with vascular invasion. At the early stage of pancreatic cancer, tumor cells have spread through the small blood vessels of the pancreas to the systemic circulation, that is, the spread of tumor cells occurs before the large blood vessels invade [18]. It is thus recognized that the cancer of the head of the pancreas involves the mesenteric or $\mathrm{PV}$, which is mainly related to the site of its occurrence. Venous involvement should not be a contraindication for surgical resection.

In conclusion, arterial priority approach for pancreatic total mesenteric resection of PD in the treatment of pancreatic head cancer can reduce intra-operative blood loss and save operation time without increasing postoperative complications. It can be used for venous system invasion of pancreatic head cancer. Combined vascular anastomosis provides effective technical support to ensure safe operation, and this procedure has improved the $\mathrm{R} 0$ resection rate of surgery and reduced the recurrence rate. Our findings indicated that it is a safe, effective and feasible surgical method for the treatment of pancreatic head cancer and is expected to be widely used in clinical surgery.

\section{Acknowledgments}

We sincerely thank the Imaging Department, Anesthesiology Department, Pathology Department and other relevant departments for their cooperation.

\section{Financial Disclosure}

This study is supported by the science and technology planning project of Weifang, Shandong province.

\section{Conflict of Interest}

All authors have no conflict of interest to declare.

\section{Informed Consent}

We obtained informed consent from all subjects and license from ethical committee.

\section{Author Contributions}

FD is responsible for experimental design and design research; $\mathrm{XW}$ and $\mathrm{XZ}$ consulted relevant literature; FD and HL performed the operation; XW collected and analyzed experimental data and wrote the paper. All the authors read and approved the manuscript.

\section{Abbreviations}

PD: pancreaticoduodenectomy; TMpE: total mesopancreas excision; BF: biliary fistula; REB: Research Ethics Board; PF: pancreatic fistula; DGE: delayed gastric emptying; CA: carbohydrate antigen; AC: abdominal cavity; AA: abdominal aorta; IVC: inferior vena cava; SMA: superior mesenteric artery; SMV: superior mesenteric vein; PV: portal vein; SEM: standard error of mean; NCCN: National Comprehensive Cancer Network; CT: coeliacus truncus

\section{References}

1. Tajima H, Ohta T, Okazaki M, Yamaguchi T, Ohbatake Y, Okamoto K, Nakanuma S, et al. Neoadjuvant chemo- 
therapy with gemcitabine-based regimens improves the prognosis of node positive resectable pancreatic head cancer. Mol Clin Oncol. 2019;11(2):157-166.

2. Leng KM, Zhong XY, Tai S, Kang PC, Wan M, Jiang XM, Wang $\mathrm{H}$, et al. Radical modular pancreatoduodenectomy for pancreatic head cancer using a combination of multiple artery-first approaches technique. Medicine (Baltimore). 2019;98(13):e14976.

3. Peery AF, Crockett SD, Barritt AS, Dellon ES, Eluri S, Gangarosa LM, Jensen ET, et al. Burden of Gastrointestinal, Liver, and Pancreatic Diseases in the United States. Gastroenterology. 2015;149(7):1731-1741 e11733.

4. Wagner M, Redaelli C, Lietz M, Seiler CA, Friess H, Buchler MW. Curative resection is the single most important factor determining outcome in patients with pancreatic adenocarcinoma. Br J Surg. 2004;91(5):586-594.

5. Huang Zhiqiang. Abdominal surgery. Hunan Science and Technology Press. 2001:1217-1228.

6. Dumitrascu T, David L, Popescu I. Posterior versus standard approach in pancreatoduodenectomy: a case-match study. Langenbecks Arch Surg. 2010;395(6):677-684.

7. Gall TM, Jacob J, Frampton AE, Krell J, Kyriakides C, Castellano L, Stebbing J, et al. Reduced dissemination of circulating tumor cells with no-touch isolation surgical technique in patients with pancreatic cancer. JAMA Surg. 2014;149(5):482-485.

8. Chen W, Zheng R, Baade PD, Zhang S, Zeng H, Bray F, Jemal A, et al. Cancer statistics in China, 2015. CA Cancer J Clin. 2016;66(2):115-132.

9. Schmidt CM, Powell ES, Yiannoutsos CT, Howard TJ, Wiebke EA, Wiesenauer CA, Baumgardner JA, et al. Pancreaticoduodenectomy: a 20-year experience in 516 patients. Arch Surg. 2004;139(7):718-725; discussion 725-717.

10. Riediger H, Makowiec F, Fischer E, Adam U, Hopt UT. Postoperative morbidity and long-term survival after pancreaticoduodenectomy with superior mesenterico-portal vein resection. J Gastrointest Surg. 2006;10(8):1106-1115. 11. Choe YM, Lee KY, Oh CA, Lee JB, Choi SK, Hur YS, Kim SJ, et al. Risk factors affecting pancreatic fistulas after pancreaticoduodenectomy. World J Gastroenterol. 2008;14(45):6970-6974.

12. Weitz J, Rahbari N, Koch M, Buchler MW. The "artery first" approach for resection of pancreatic head cancer. J Am Coll Surg. 2010;210(2):e1-4.

13. Ravikumar R, Sabin C, Abu Hilal M, Bramhall S, White $\mathrm{S}$, Wigmore S, Imber CJ, et al. Portal vein resection in borderline resectable pancreatic cancer: a United Kingdom multicenter study. J Am Coll Surg. 2014;218(3):401411.

14. Zhou Y, Zhang Z, Liu Y, Li B, Xu D. Pancreatectomy combined with superior mesenteric vein-portal vein resection for pancreatic cancer: a meta-analysis. World J Surg. 2012;36(4):884-891.

15. Wang C, Wu H, Xiong J, Zhou F, Tao J, Liu T, Zhao G, et al. Pancreaticoduodenectomy with vascular resection for local advanced pancreatic head cancer: a single center retrospective study. J Gastrointest Surg. 2008;12(12):21832190.

16. Yekebas EF, Bogoevski D, Cataldegirmen G, Kunze C, Marx A, Vashist YK, Schurr PG, et al. En bloc vascular resection for locally advanced pancreatic malignancies infiltrating major blood vessels: perioperative outcome and long-term survival in 136 patients. Ann Surg. 2008;247(2):300-309.

17. Shimoda M, Mori S, Kita J, Sawada T, Kubota K. Results of pancreaticoduodenectomy with portal or superior mesenteric vein resection for locally advanced pancreatic head cancer. Hepatogastroenterology. 2013;60(128):20942098.

18. Rehders A, Stoecklein NH, Guray A, Riediger R, Alexander A, Knoefel WT. Vascular invasion in pancreatic cancer: tumor biology or tumor topography? Surgery. 2012;152(3 Suppl 1):S143-151. 